\title{
PvRACK1 Loss-of-Function Impairs Cell Expansion and Morphogenesis in Phaseolus vulgaris L. Root Nodules
}

\author{
Tania Islas-Flores, ${ }^{1}$ Gabriel Guillén, ${ }^{1}$ Xóchitl Alvarado-Affantranger, ${ }^{1}$ Miguel Lara-Flores, ${ }^{2}$ \\ Federico Sánchez, ${ }^{1}$ and Marco A. Villanueva ${ }^{3}$
}

\begin{abstract}
${ }^{1}$ Departamento de Biología Molecular de Plantas, Instituto de Biotecnología, and ²Departamento de Genómica Funcional de Eucariotes, Centro de Ciencias Genómicas, Universidad Nacional Autónoma de México (UNAM), Apartado Postal 510-3, Cuernavaca, Morelos 62250, México; ${ }^{3}$ Instituto de Ciencias del Mar y Limnología, Unidad Académica de Sistemas Arrecifales, UNAM, Prolongación Avenida Niños Héroes S/N, Puerto Morelos, Quintana Roo 77580, México
\end{abstract}

Submitted 13 November 2010. Accepted 11 March 2011.

\begin{abstract}
Receptor for activated C kinase (RACK1) is a highly conserved, eukaryotic protein of the WD-40 repeat family. Its peculiar $\beta$-propeller structure allows its interaction with multiple proteins in various plant signal-transduction pathways, including those arising from hormone responses, development, and environmental stress. During Phaseolus vulgaris root development, RACK1 (PvRACK1) mRNA expression was induced by auxins, abscissic acid, cytokinin, and gibberellic acid. In addition, during $P$. vulgaris nodule development, PvRACK1 mRNA was highly accumulated at 12 to 15 days postinoculation, suggesting an important role after nodule meristem initiation and Rhizobium nodule infection. PvRACK1 transcript accumulation was downregulated by a specific RNA interference construct which was expressed in transgenic roots of composite plants of $P$. vulgaris inoculated with Rhizobium tropici. PvRACK1 downregulated transcript levels were monitored by quantitative reverse-transcription polymerase chain reaction analysis in individual transgenic roots and nodules. We observed a clear phenotype in PvRACK1-knockdown nodules, in which nodule number and nodule cell expansion were impaired, resulting in altered nodule size. Microscopic analysis indicated that, in PvRACK1-knockdown nodules, infected and uninfected cells were considerably smaller $(80$ and $60 \%$, respectively) than in control nodules. In addition, noninfected cells and symbiosomes in silenced nodules showed significant defects in membrane structure under electron microscopy analysis. These findings indicate that PvRACK1 has a pivotal role in cell expansion and in symbiosome and bacteroid integrity during nodule development.
\end{abstract}

Eukaryotic cells have the ability to integrate signals using adaptor proteins that allow the formation of multiprotein complexes with signaling components in proximity. These adapter proteins play key roles in the multiple signal-transduction processes that normally occur within a cell. The so-called $\beta$ propeller structure is a molecular platform capable of integrating several signals because many proteins can display the

Corresponding author: M. A. Villanueva; Telephone: +52 (998) 8710219 or +52 (998) 8710009 ext. 177; Fax: +52 (998) 871 0138; E-mail: marco@cmarl.unam.mx

This article is dedicated to the memory of our dearest colleague, Carolina San Román-Roque, who made this research possible and passed away on 12 March 2011. structure even though they play diverse biological functions and, at the same time, multiple proteins can interact with such structure. Receptor for activated C kinase (RACK1), which is a highly conserved scaffold protein found ubiquitously expressed in both animals and plants, is a member of this family of proteins and contains seven WD-40 repeats that form its $\beta$ propeller structure. Although it was originally identified as an anchoring protein for activated protein kinase $\mathrm{C}$ (PKC) in mammals (Ron et al. 1994), it is now recognized as a versatile scaffold protein that can bind several signaling molecules from diverse cellular pathways (McCahill et al. 2002). In mammalian cells, RACK1 participates in diverse biological processes such as cell growth (McCahill et al. 2002), assembly of the $80 \mathrm{~S}$ ribosome from $40 \mathrm{~S}$ and 60S subunits (Ceci et al. 2003), and neuropathology, cellular stress, protein translation, and developmental processes (Ullah et al. 2008), among others. In addition, RACK1 is overregulated during angiogenesis and in human carcinoma, and can contribute to growth and tumor spreading (Berns et al. 2000).

Plant RACK1 was first identified as the product of an auxinregulated gene from cultured cells (arcA) isolated from a differentially expressed library to screen auxin-responsive genes in tobacco BY2 cells (Ishida et al. 1993). To date, cDNA homologous sequences have been identified in a number of plants, including soybean (G protein $\beta$-like $[G \beta$-like]; Nielsen et al. 2001); alfalfa (Medicago sativa G $\beta$-like [Msgb1]; McKhann et al. 1997), Arabidopsis thaliana (AtarcA), rice (rice protein containing the WD-40 repeat [RWD]; Iwasaki et al. 1995, Nakashima et al. 2008), tomato (LeArcA; Kiyosue and Ryan 1999), Brassica napus (Brassica G protein $\beta$ subunit 1 [BGB1]; Kwak et al. 1997), and common bean (Phaseolus vulgaris [PvRACK1]; Islas-Flores et al. 2009). Recently, it was reported that, in the rice genome, two RACK1 genes (RACK1A and $R A C K 1 B)$ are encoded, and that one of them $(R A C K 1 A)$ is positively regulated by Rac1, auxin, abscissic acid (ABA), and jasmonate (Nakashima et al. 2008). When Oryzae sativa RACK1A expression was silenced by RNA interference (RNAi), the regeneration of viable plants from rice callus, production of $\mathrm{H}_{2} \mathrm{O}_{2}$, and expression of the pathogen defense gene, $P B Z 1$, greatly decreased. Thus, $O$. sativa $R A C K 1 A$ also appears to play an important role in reactive oxygen species production and defense gene expression in rice cell cultures (Nakashima et al. 2008). In legume roots, this protein has been implicated in hormone-mediated signal transduction; for example, the $M$. sativa Msgbl (RACK1A homologous gene) was induced when alfalfa roots were treated with cytokinin. In addition, a high level of accumulation was observed in nodule 
meristem after Sinorhizobium meliloti inoculation (McKhann et al. 1997). During nodule formation in plants, there is active cell growth and division in a highly proliferating and regulated process. This could be regarded as similar to processes occurring during growth and proliferation in mammalian cells, where RACK1 is involved. Therefore, the nodulation process is a provoking model for studying RACK1 function in plants. Prior to this nodulation process, a specific recognition of the bacteria by the plant and a molecular dialogue between Nod factors from Rhizobiaceae bacteria and the plant root receptors must occur. Several signaling molecules involved in Nod factor perception have been identified, including receptor-like kinases (Gough 2003). In addition, the Nod factor signaling events are most likely transduced by a signaling cascade implicating adaptor proteins and second messengers. Considering that i) the nitrogen-fixing root nodule is a plant organ where an intense program of cell proliferation and expansion occurs (Crespi and Frugier 2008), ii) PvRACK1 has biochemical features similar to other RACK1 proteins (36-kDa protein, seven WD-40 repeats, and conserved PKC-binding domains), and iii)
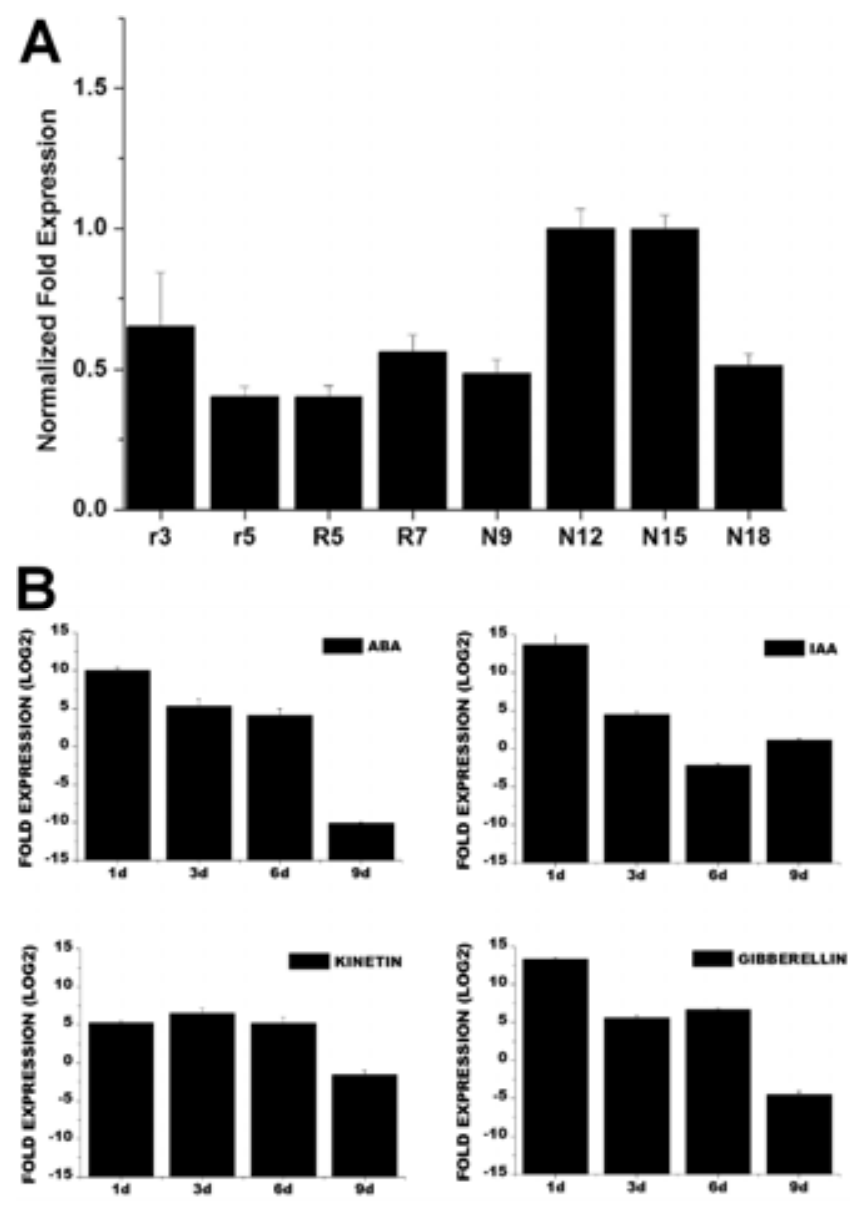

Fig. 1. Comparative expression analysis of $\mathbf{A}$, the Phaseolus vulgaris receptor for activated $\mathrm{C}$ kinase $(P v R A C K 1)$ gene throughout an 18-day nodulation kinetics and $\mathbf{B}$, the effect of hormones on the PvRACK1 gene during root development. A, Amplification by quantitative reverse-transcription polymerase chain reaction (qRT-PCR) was performed in triplicates and normalized to the average of one reference gene (PvIEF1). Quantified RNA was analyzed after harvesting from uninoculated roots (r), postinoculated roots $(\mathrm{R})$, and root nodules $(\mathrm{N})$ at the indicated number of days. B, $P$. vulgaris seedlings were treated with $10 \mathrm{mM}$ abscisic acid (ABA), $25 \mathrm{mM}$ indole-3-acetic acid (IAA), $25 \mathrm{mM}$ kinetin, or $10 \mathrm{mM}$ gibberellic acid $\left(\mathrm{GA}_{4}\right)$ for $1,3,6$, and 9 days. Total RNA was extracted and analyzed by qRT-PCR. Expression levels of PvRACK1 were normalized to PvIEF1. Values represent the normalized +hormone or -hormone fold expression as the average of three replicates ( $\log _{2}$-converted).
PvRACK1 is very likely encoded by a single gene in the common bean genome (Islas-Flores et al. 2009), we searched for the possible involvement of PvRACK1 in root nodule development by RNAi. We report here that PvRACK1-knockdown common bean nodules have a strong nodulation phenotype in which nodule cell expansion and symbiosome and bacteroid integrity are severely affected.

\section{RESULTS}

$P v R A C K 1$ is differentially expressed during nodulation.

RACK1 is a protein that participates in diverse plant developmental processes (Guo and Chen 2008). Therefore, despite its ubiquitous expression, changes in expression can be expected in different cellular types and physiological stages.

In order to confirm our prediction that PvRACK1 could participate in proliferation processes associated with $P$. vulgaris nodules, we quantified mRNA levels of PvRACK1 during nodule development. mRNA levels increased slowly after inoculation with Rhizobium tropici (Fig. 1A, R5 to N18) and reached a peak of highest accumulation at 12 to 15 days postinoculation (dpi) (Fig. 1A, N12 to N15). These time points correspond, in particular, to those developmental stages in which determinate nodule meristem cells cease to divide (nodule meristem exhausts) and cells continue to grow by cell expansion. Therefore, these results indicate that PvRACK1 is mostly accumulated at the onset of intracellular rhizobia infection and nodule cell expansion.

\section{PvRACK1 expression is induced} by indole-3-acetic acid, ABA, cytokinin, and gibberellin.

Plant RACK1 homologs have been shown to be involved in hormonal responses (Chen et al. 2006; Guo et al. 2009; Ishida et al. 1993; Iwasaki et al. 1995; Kwak et al. 1997; McKhann et al. 1997; Nakashima et al. 2008). We reported previously that PvRACK1 expression is not severely affected by indole-3-acetic acid (IAA) in early germination (Islas-Flores et al. 2009); however, nothing was known about the effect of other hormones on PvRACK1 expression in other tissues. Therefore, we tested the effect of auxin (25 $\mu \mathrm{M}$ IAA), ABA $(10 \mu \mathrm{M})$, cytokinin $(25$ $\mu \mathrm{M}$ kinetin), and gibberellic acid $(10 \mu \mathrm{M} \mathrm{GA} 4)$ on PvRACK1 expression in roots after 1, 3, 6, and 9 days of treatment (Fig. 1B). All these hormones resulted in the induction of PvRACK1 expression from the first day of treatment and in a clear decrease after 3 to 9 days of treatment for ABA, IAA, and GA. Only with the cytokinin treatment did the induced expression level remain relatively constant (Fig. 1B). These findings indicate that PvRACK1 expression is also modulated by hormonal levels during root development in $P$. vulgaris.

\section{PvRACK1 silencing phenotype.}

The scaffold function of RACK1 suggests a role in multiple signal transduction and hormonal signaling processes in a regulated fashion. Thus, to maintain the cellular balance, its regulation must be finely controlled. To analyze PvRACK1 function in $P$. vulgaris nodulation, and with several lines of evidence suggesting that it is most likely represented by a single gene in the $P$. vulgaris genome (Islas-Flores et al. 2009), a specific PvRACK1-RNAi construction was made and transformed into composite common bean plants to knock down PvRACK1 expression. After PvRACK1 silencing (approximately 90\%) (Fig. 2D), the transgenic silenced roots of composite plants showed a similar phenotype (Fig. 2C) compared with control roots (Fig. 2A and B). However, the nodules of transgenic composite plants were observed to be smaller in size, with a whitish color, and, moreover, reduced in number (Fig. 2C and D) with respect to control plants (Fig. 2A, B, and D). In order to make 
sure that the PvRACK1 mRNA expression was inhibited, the transcript level was quantified by real-time reverse-transcription polymerase chain reaction (RT-PCR) in individual roots (Fig. 2D, solid bars) and nodules (Fig. 2D, empty bars) from control and PvRACK1-knockdown composite plants (Fig. 2D; K599, EMPTY, P4RNAiR1, P6RNAiR1, P6RNAiR2). The silencing level obtained was up to approximately $90 \%$, and the results indicate that the phenotype is due to this low level of PvRACK1 mRNA expression. The developmental stage of the PvRACK1-knockdown nodules indicates a defect in nodule growth, and is reminiscent of the developmental stage of a nodule in which growth by cell expansion has not yet occurred. At this developmental stage, PvRACK1 mRNA accumulation levels in a normal nodule correspond to those observed from 5 to 18 dpi (Fig. 1, R5-N18). These results clearly demonstrate that PvRACK1 knockdown severely affects the nodulation process by altering its morphology and number.

\section{PvRACK1 silencing results}

\section{in small whitish nodules with reduced cell sizes.}

Silencing of PvRACK1 expression affects the nodule size (Fig. 2C) but, to obtain more detailed information about this phenotype in terms of cell structure and morphology, a microscopic analysis of the silenced nodules was carried out. A microscopic analysis from control and PvRACK1-knockdown nodules showed a similar number and distribution of infected and uninfected cells in silenced nodules (Fig. 3C and D and inset in C) with respect to control (Fig. 3A and B and inset in A). However, it was immediately obvious that the cell size of the nodule central zone was reduced, and the cortex was enlarged and greatly disorganized in the PvRACK1-knockdown nodules (Fig. 3C and D) compared with control nodules (Fig. 3A and B). The cell size was scored in various samples and sections, and a bar graph was made to show the differences in dimension of both infected and uninfected cells from PvRACK1-knockdown and control nodules (Fig. 4). The larger differences were found between infected cells from PvRACK1knockdown nodules (Fig. 4, RNAi, empty bars) compared with those of K599 control nodules (Fig. 4, K599, empty bars). Differences in size were statistically significant for both infected and uninfected cells, thus suggesting that the reduction in cell size arose from the silencing of PvRACK1 expression.

\section{Electron microscopy of PvRACK1-knockdown nodules.}

To obtain more information about the effect of PvRACK1 silencing at the subcellular level, an ultrastructural analysis by electron microscopy of PvRACK1-knockdown and control nodules was made. Electron micrographs showed evident distinctive features in the PvRACK1-knockdown infected cells. Control infected and uninfected cells were regular in size and structure (Fig. 5A to D), with abundant and normal bacteroids (B in Fig. $5 \mathrm{~A}, 5 \mathrm{C}$, and 5D) enclosed by intact-looking symbiosomes (Fig. 5C), with a complete and delimited peribacteroidal membrane (Fig. 5C, peribacteroid membrane [PBM], arrows). In addition, the bacteroids had numerous polyhydroxybutyrate granules (Fig. $5 \mathrm{D}, \mathrm{PHB})$. However, the most evident difference in the infected cells of PvRACK1-knockdown nodules (Fig. 5E to H), was the abnormal structure of the bacteroids, with almost no polyhy-
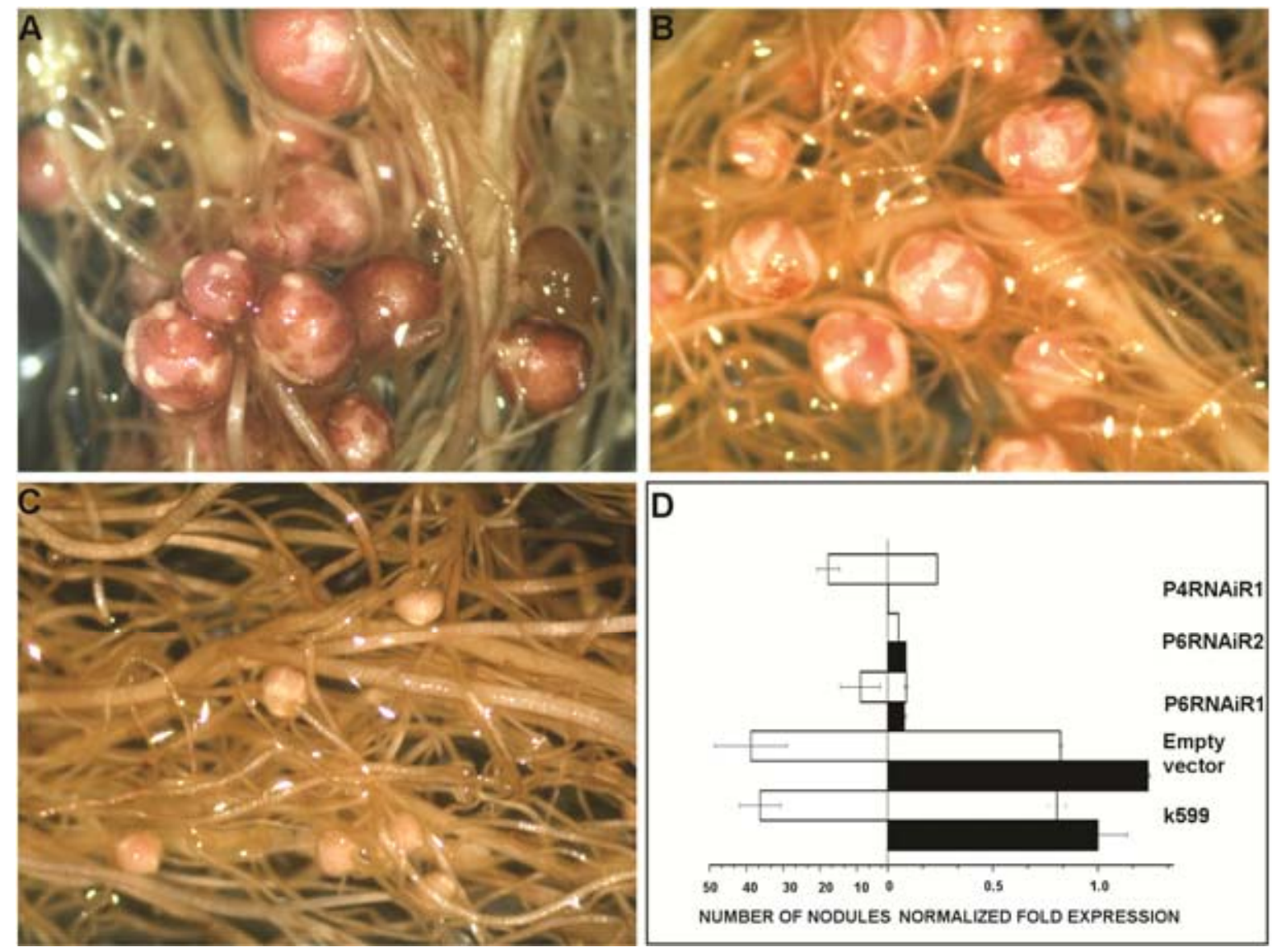

Fig. 2. Phaseolus vulgaris receptor for activated $\mathrm{C}$ kinase ( $P v R A C K 1)$-knockdown phenotype. $\mathbf{C}$, Examples of morphological phenotypes of root nodules resulting from PvRACK1-knockdown compared with $\mathbf{A}$, the wild type and $\mathbf{B}$, control. D, Nodule number (left side of graphic) and real-time reverse-transcription polymerase chain reaction (RT-PCR) analysis (right side of graphic) from mRNA levels when PvRACK1-RNA interference (RNAi) was expressed (P4RNAiR1, P6RNAiR1, P6RNAiR2) in individual transgenic roots (solid bar) and nodules (empty bar) and compared with those of hairy root (K599) and control (empty vector). Amplification by real-time RT-PCR was performed in triplicate and normalized to the average of one reference gene (PvIEF1). 
droxybutyrate granules (B in Fig. $5 \mathrm{G}$ and $\mathrm{H}$ ), with many of these bacteroids collapsed (Fig. 5G) or in the process of disintegration (Fig. 5H). Interestingly, bacteroid size was similar in knockdown and control nodules (Fig. 5G and $\mathrm{H}$ compared with $\mathrm{C}$ and $\mathrm{D})$. In addition, the cytoplasmic and symbiosomal matrix of control nodules showed an electron-dense material that formed a regularly organized network pervading them (Fig. 5D). In contrast, PvRACK1-knockdown infected cells were disorganized and denser (Fig. 5E to $\mathrm{H}$ ), and this electron-dense matrix was severely disorganized (Fig. 5H). These findings indicate that PvRACK1 knockdown also has a profound negative effect on the symbiosome and bacteroid organization and integrity as well as the cytoplasmic matrix.

\section{DISCUSSION}

RACK1 is a versatile protein involved in several cellular processes. The seven-blade $\beta$-propeller structure renders the PvRACK1 protein capable of interacting simultaneously with several signaling molecules in a regulated fashion. Plant RACK1 homologs have been shown to play an important role in hormone signaling, development, and innate immunity (Chen et al. 2006; Nakashima et al. 2008); however, RACK1 function in plants is not yet fully understood.

Nodule and tumor growth resemble each other in terms of angiogenesis formation and the requirement of active prolif- eration. Indeed, mammalian RACK1 has been shown to be important for the maintenance of tumor angiogenesis and tumor growth. It is upregulated in different angiogenically active tissues, both in vitro and in vivo. In addition, it is expressed in tumor angiogenesis (Berns et al. 2000) and overexpressed in colon cancer cells (Saito et al. 2002). RACK1 also promotes breast carcinoma proliferation and invasion or metastasis in vitro and in vivo, and knockdown of RACK1 in breast carcinoma and in LNCap cells inhibits cell proliferation and tumor growth (Cao et al. 2009, Kraus et al. 2006). Consistent with our hypothesis of PvRACK1 participating in cell proliferationrelated processes, we found that PvRACK1 mRNA was differentially expressed during nodulation. The increased accumulation of PvRACK1 mRNA in nodules corresponding to 12 to 15 dpi (Fig. 1A, N12 to N15) showed a potential involvement of this protein in nodule growth. Furthermore, we observed that downregulation of PvRACK1 by RNAi in transgenic roots of composite plants produced a striking phenotype in which nodule number was decreased to 50 to $76 \%$ less nodules than control, and growth was reduced. This phenotype is consistent with that reported for A. thaliana RACK1A loss-of-function, which showed developmental defects in seedlings and mature plants (shorter hypocotyls and reduced rate of rosette leaf production) (Chen et al. 2006), although a microscopic assessment of cellular structures was not carried out in that study. During nodule ontogeny, hormones play important roles regulating nod-
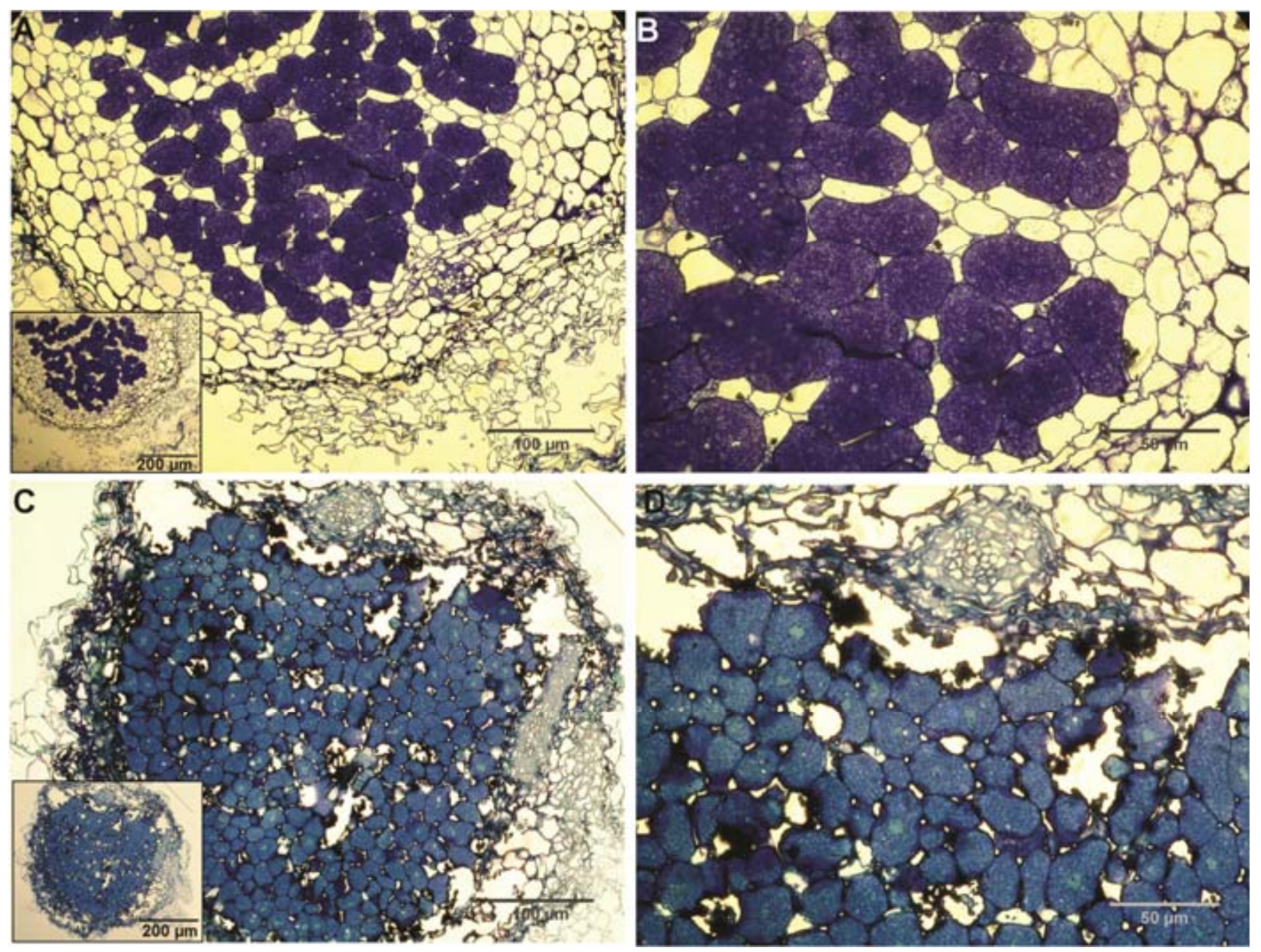

Fig. 3. Conventional light microscopy of structural features from Phaseolus vulgaris receptor for activated C kinase (PvRACK1)-knockdown and control nodules at 26 days postinoculation. Light micrographs were taken from microtome slices prepared from C, inset, and D, PvRACK1-knockdown and A, inset, and $\mathbf{B}$, control nodules and stained with toluidine blue. 
ule morphogenesis and development. Auxin, ABA, cytokinin, and GA can be isolated from nodules, where they are in higher concentrations than in uninfected roots (Hirsch and Fang 1994), indicating a pivotal role regulating nodule morphogenesis and function. Auxins, cytokinin, and GA are positive regulators of nodulation. Signaling by these hormones was affected by the expression of RACK1 in Arabidopsis (Chen et al. 2006). The knockout rackla mutant of $A$. thaliana showed altered sensitivities to hormones; that is, less sensitive to GA and brassinosteroids (BR) and more responsive to ABA in seed germination and early seedling development, and less responsive to auxin, leading to reduced root size (Chen et al. 2006). These data are consistent with the previously reported roles of RACK1 in hormonal signal transduction (Ishida et al. 1993; Iwasaki et al. 1995; Kwak et al. 1997; McKhann et al. 1997). For example, in $M$. sativa, the $M s g b l$ gene ( $R A C K 1$ homolog) was induced when alfalfa roots were treated with cytokinin. In addition, a high level of accumulation was observed in nodule meristem after S. meliloti inoculation (McKhann et al. 1997), suggesting an important role of Msgb1 in nodulation of this plant. In this work, we also found that PvRACK1 expression is modulated by the presence of the hormones IAA, ABA, cytokinin, and gibberellins during root development (Fig. 1B). This, in turn, most likely reflects the multiple levels of regulation and mutual interaction of hormones and PvRACK1 expression during nodulation, which is highly consistent with the PvRACK1knockdown phenotype. The reduced cell size arising from PvRACK1-knockdown nodules suggests an effect in cell expansion that might be a result of the impairment of hormonemediated signaling. (Ding and Oldroyd 2009; Oldroyd and Downie 2008). Thus, one might speculate that the involvement of plant RACK1 homologs in hormone signaling might be one of the keys to the silencing phenotype in $P$. vulgaris nodulation (less nodule production and smaller nodules) (Fig. 2C). In the knockout, the lack of PvRACK1 expression might produce less sensitivity to GA and BR, that are positive regulators of nodule morphogenesis (Ding and Oldroyd 2009), and to ABA, which is a negative regulator and is, at the same time, negatively regulated by RACK1A and RACK1C as found in Arabidopsis (Guo et al. 2009). In addition, the appearance of a small number of nodules could indicate an effect of PvRACK1 in an early nodulation stage such as infection; this idea is supported by the fact that we have also observed an increase in PvRACK1 mRNA by quantitative RT-PCR after the first $2 \mathrm{~h}$ postinoculation (data not shown). Although we cannot conclude that the effect of PvRACK1 knockdown is nodule specific, the fact that the PvRACK1 knockdown roots do not seem to be affected suggests that there are different levels of regulation in cell expansion of these organs. Another pathway that could be possibly affected by PvRACK1 silencing is membrane synthesis, trafficking, and recycling, which is important for growth by cell expansion. In a previous report, srabl and vrab7 silencing in soybean resulted in smaller nodules and, when srabl was silenced, fewer nodules were formed (Cheon et al. 1993). Plant Rab1p and Rab7p are small GTP-binding proteins involved in the biogenesis of the PBM (Cheon et al. 1993); therefore, an effect on these proteins could severely affect membrane dynamics and, consequently, nodule size. These results are also consistent with the phenotype provoked by PvRACK1 silencing on common bean. Thus, another possibility is that PvRACK1 is also important for membrane dynamics and its silencing may result in an additive effect of both interference on membrane trafficking and hormone-mediated signaling on nodule development and integrity. Vesicular traffic is necessary during

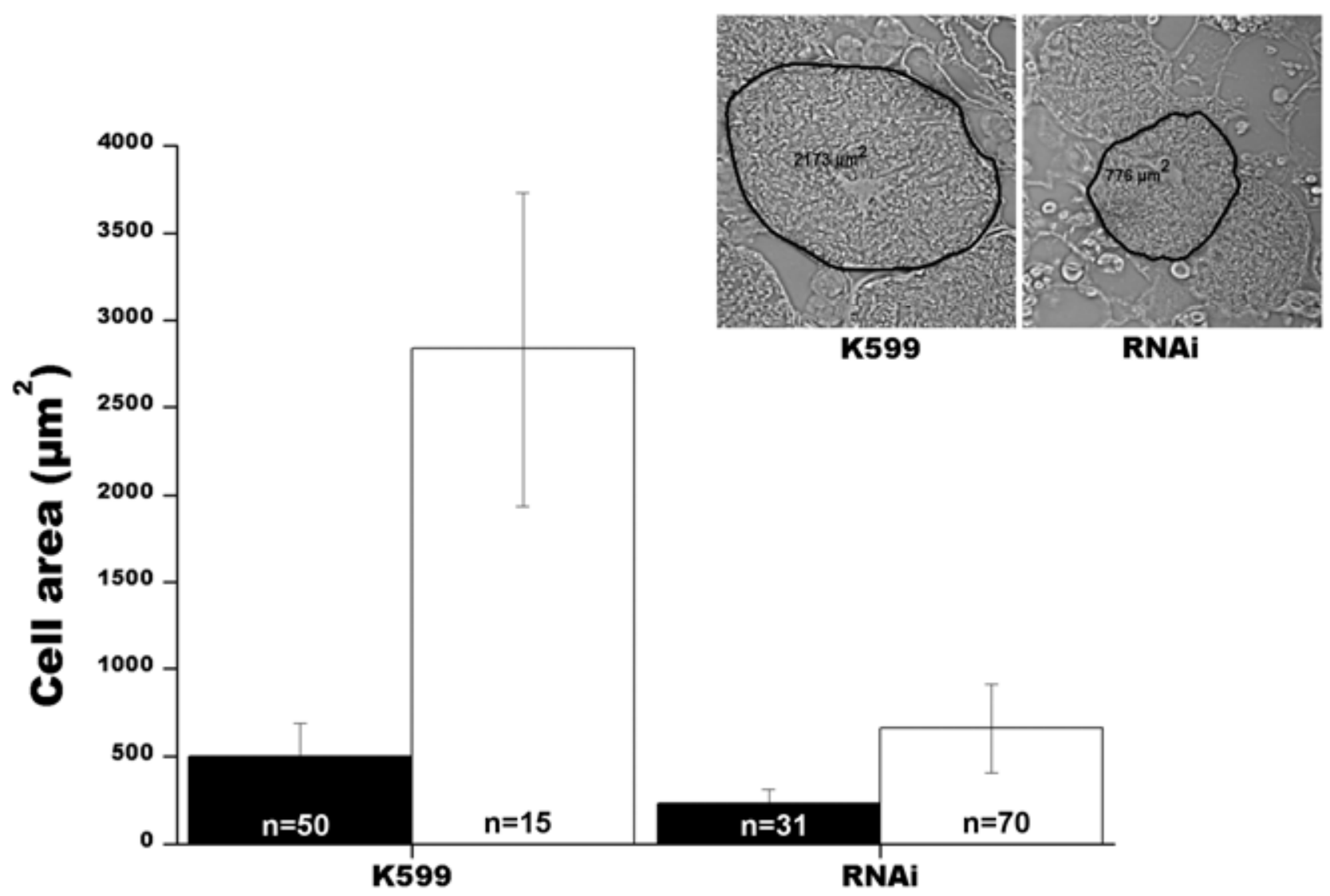

Fig. 4. Quantification of cell size in infected and uninfected cells from control (K599) and Phaseolus vulgaris receptor for activated C kinase (PvRACK1)knockdown nodules. Graph shows the cell area of infected (empty bars) and uninfected (solid bars) cells. Insets show the areas from representative cells from control infected (K599) and PvRACK1-knockdown nodules; $n=$ number of measured cells in sample and area was measured in square micrometers. 
membrane synthesis for cell expansion. Therefore, its deregulation would severely affect such membrane synthesis at key sites. This would, in turn, produce phenotypes with altered or defective membranes, which will result in a smaller size of both infected and uninfected cells (Figs. 3 and 4) and abnormal PBM in symbiosomes (Fig. 5G and H), and in the symbiosome cytoplasmic matrix surrounding the bacteroids. The involvement of mammalian RACK1 in these vesicular traffic and endosome recycling processes could occur at the level of interaction with one of its binding partners, PI3K. For example, it has been reported that PI3K has a fundamental role in Rab11 localization during mitosis in Caenorhabditis elegans (Ai et al. 2009; Chen et al. 2008; McCahill et al. 2002). Alternatively, based on the deteriorated appearance of the knockdown nodules, an additional effect of PvRACK1 silencing could be the induction of early senescence of the nodule. A key to clarifying the level of these interactions will come from studies on RACK1 ligands in plants. To date, there is no information whatsoever about RACK1 binding partners in this kingdom.

The data obtained here indicate that PvRACK1 plays a pivotal role in nodule growth and maintenance because deteriorated infected cells and bacteroids of PvRACK1-knockdown nodules (Fig. 5E to $\mathrm{H}$ ) are also indicative of an impaired nodule function. The phenotype at the ultrastructural level is also similar to that of Lotus japonicus ign1 mutant. IGN1 belongs to an ankyrin repeat protein family, in which symbiosomes are enlarged relative to their bacteroid number (Kumagai et al. 2007).
RACK1 is known to be a versatile protein with many binding partners, which make it prone to be involved in many cellular functions. This fact suggests that it is tightly regulated and its involvement in a single specific function is very difficult to assign. For this reason, various cellular functions are probably affected when its expression is perturbed. Regardless of the specific PvRACK1 functions that lead to a normal nodule development in common bean, in this work we have clearly demonstrated that it is indispensable for symbiosome integrity, bacteroid development, and nodule expansion in infected and uninfected cells in this species.

\section{MATERIALS AND METHODS}

\section{Plant and cell lines.}

P. vulgaris L. 'Negro Jamapa' seed were used. Seed were surface-sterilized with chloride gas in a fume hood for $16 \mathrm{~h}$ before germination. Agrobacterium rhizogenes K599 was used for root transformation and $R$. tropici CIAT899 were used to induce the nodulation.

\section{Hormone treatment.}

Surface-sterilized seed of Negro Jamapa were germinated in wet, sterile towels for 4 days; then, seedlings were transferred to a hydroponic medium with $\mathrm{B} \& \mathrm{D}$ solution supplemented with nitrogen (Estrada-Navarrete et al. 2007) at 26 to $28^{\circ} \mathrm{C}$ for 3 days. After this period, hormones were added $(25 \mu \mathrm{M}$ IAA, $10 \mu \mathrm{M}$ ABA, $25 \mu \mathrm{M}$ kinetin, and $10 \mu \mathrm{M} \mathrm{GA}_{4}$ ). Roots were col-
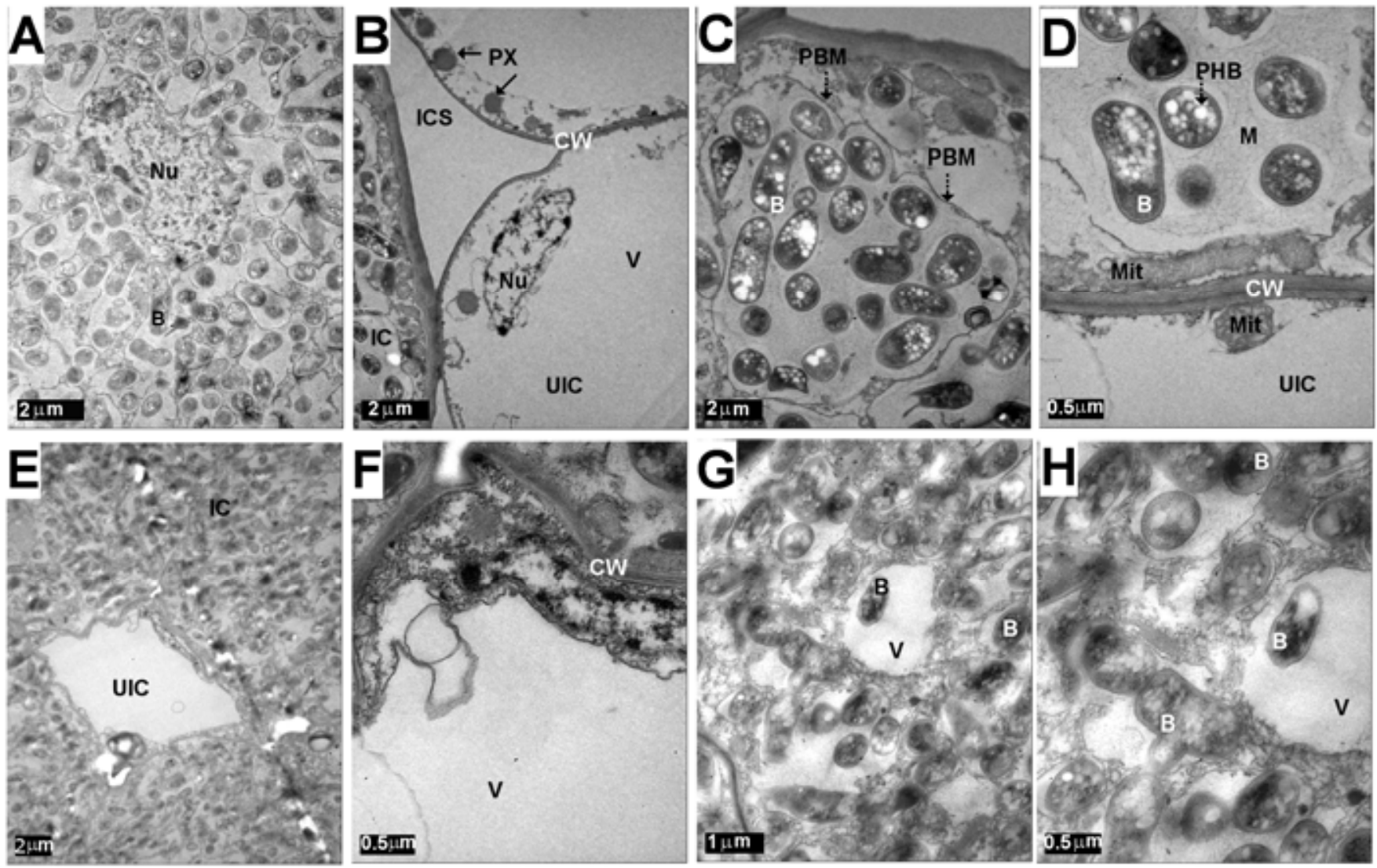

Fig. 5. Ultrastructural features of control and Phaseolus vulgaris receptor for activated $\mathrm{C}$ kinase (PvRACK1)-knockdown nodules by electron microscopy. A to $\mathbf{D}$, Control nodules, 26 days postinoculation (dpi). $\mathbf{E}$ to $\mathbf{H}$, PvRACK1-knockdown nodules, 26 dpi. A, Nucleus (Nu) and $\mathbf{B}$, bacteroids of an infected cell are shown. C, Peroxisomes (PX; solid line arrows), nucleus, vacuole (V), and cell wall (CW) of uninfected cells (UIC) are clearly observed, as well as the intracellular space (ICS) and an adjacent infected cell (IC). C, Dashed line arrows depict the peribacteroid membrane (PBM). D, Bacteroids (B), matrix (M), a bacteroid with a polyhydroxybutyrate granule (PHB), and mitochondria (Mit) are clearly distinguished. On the other hand, in PvRACK1-knockdown nodules, $\mathbf{E}$ and $\mathbf{F}$, both uninfected and infected cells show lack of integrity and $\mathbf{G}$ and $\mathbf{H}$, bacteroids are in the process of disintegration. $\mathrm{H}$ shows the magnified central area of G. 
lected at 1, 3, 6, and 9 days after application of the hormone treatment and stored at $-70^{\circ} \mathrm{C}$. Total RNA was extracted with the hot phenol method with some modifications (Pawlowski et al. 1994), and RNA concentration was determined with a NANODROP 2000c spectrophotometer (Thermo Fisher Scientific, Waltham, MA, U.S.A.).

\section{PvRACK1 silencing constructs.}

Total RNA was isolated from nodules $15 \mathrm{dpi}$ by the Chomczynski and Sacchi (1987) method, with some modifications. The PvRACK1 cDNA sequence for RNAi was amplified with the primers $5^{\prime}$-CACCATGGCGGAAGGACTCGTCCTG AAGG-3' (forward) and 5'-TACGGTCCCAAGATGCA-3' (reverse). cDNA fragments of $520 \mathrm{bp}$ were cloned into the pENTR/ D-TOPO vector (Invitrogen, Carlsbad, CA, U.S.A.) and sequenced, and the resulting plasmids were recombined with the destiny vector pTDT-DC-RNAi (Valdés-López et al. 2008). The pTDT-PvRACK1-RNAi construct was generated.

\section{A. rhizogenes induction of $P$. vulgaris composite plants.}

Surface-sterilized seed of Negro Jamapa were germinated in wet sterile towels for 3 days; then, seedlings were inoculated with A. rhizogenes K599 either alone or carrying the silencing construction pTDT-PvRACK1-RNAi in the upper side of the root. They were then transferred to a hydroponic chamber with B\&D-supplemented solution (Estrada-Navarrete et al. 2007) without nitrogen and appropriated aeration at 26 to $28^{\circ} \mathrm{C}$. After 3 days, $R$. tropici CIAT899 was added to the solution and, 26 days after, nodulated hairy roots were analyzed by microscopy. Nodules were collected and processed for microscopic analysis or frozen in liquid nitrogen and stored at $-80^{\circ} \mathrm{C}$ until analysis.

\section{Real-time PCR and RT-PCR quantification analyses.}

For the quantitative analysis of the PvRACK1 transcript level, total RNA was extracted from silenced and control roots and nodules and hormone-treated roots. The RNA was treated with DNaseI (Invitrogen), and the concentration was determined by absorbance at 260 and $280 \mathrm{~nm}$ in a NANODROP 2000c spectrophotometer (Thermo Scientific). For transgenic roots and nodules and the nodulation kinetics, RNA (100 ng) was then mixed with the iScript One-Step RT-PCR kit with SYBR Green (BioRad, Hercules, CA, U.S.A.). For the hormone-treated roots, cDNA was synthesized with the RevertAid H Minus FirstStrand cDNA synthesis kit (Fermentas Life Sciences, Waltham, MA, U.S.A.), and $50 \mathrm{ng}$ of cDNA was mixed with Maxima SYBR Green/Fluorescein qPCR Master Mix (Fermentas Life Sciences). Each of the PvRACK1 primers 5'-CTGCACTCCA GTTCAAACTCG-3' (forward) and 5'-GAGCAGAGCATCAA GATCTGG-3' (reverse), and elongation factor 1 (EF1) 5'-GGTC ATTGGTCATGTCGACTCTGG-3' (forward) and 5'-GCACCC AGGCATACTTGAATGACC-3' (reverse), as the control housekeeping gene, were added to each reaction at a final concentration of $10 \mu \mathrm{M}$. EF1 primer design was based on the reported bean expressed sequence tag sequence with the GenBank accession number CV530481. Quantitative real-time RT-PCR was done in triplicate reactions on a qPCR-iQ5 or a CFX96 Real Time System C1000 Thermal Cycler (Bio-Rad).

\section{Nodulation kinetics and tissue collection.}

Three-day-old seedlings were root inoculated with $R$. tropici CIAT899, and noninoculated roots or roots from 5 and 7 dpi and nodules from 9, 12, 15, and 18 dpi were collected and frozen until ready for RNA analysis.

\section{Microscopy.}

At 26 dpi, PvRACK1-knockdown and standard P. vulgaris roots and nodules infected with $R$. tropici CIAT899 were col- lected, fixed, embedded, polymerized with methacrylate, sectioned, and rehydrated as previously reported (Estrada-Navarrete et al. 2006). After rehydration, root and nodule sections were mounted on a slide and a coverslip for observation under a Zeiss Axiovert $200 \mathrm{M}$ microscope. Images were processed using Adobe Photoshop 7.0 software (Adobe Systems Inc., Mountain View, CA, U.S.A.).

\section{Electron microscopy.}

Nodule samples were cut in 1- to 2-mm sections, fixed, dehydrated (30 to $100 \%$ ethanol series), and pre-embedded in propylene oxide/epoxy resin mixtures $(2: 1,1: 1$, and 1:2) (London Resin Company Limited, London) for several hours before embedding in $100 \%$ epoxy resin for $3 \mathrm{~h}$. Nodules pieces in gelatin capsules (Electron Microscopy Sciences, Fort Washington, PA, U.S.A.) were embedded in epoxy resin. Ultrathin sections $(70 \mathrm{~nm})$ were obtained with a UCT-R ultramicrotome (Leica, Wetzlar, Germany) and mounted on nickel grids. They were then stained with $2 \%$ aqueous uranyl acetate and visualized under a Zeiss EM900 transmission electron microscope at $80 \mathrm{kV}$. Micrographs were taken with a digital CCD DualVision 300W camera (Gatan, Inc., Pleasanton, CA, U.S.A.).

\section{ACKNOWLEDGMENTS}

We thank G. Zavala from the Electron Microscopy facility of the Institute of Biotechnology-UNAM for her expert technical help and the Oligonucleotide Synthesis facility of the Institute of Biotechnology-UNAM for the synthesis of specific primers. This work was supported by grant 83324 from CONACyT. T. Islas-Flores was supported by a Ph.D. scholarship (176434) from CONACyT.

\section{LITERATURE CITED}

Ai, E., Poole, D. S., and Skop, A. R. 2009. RACK-1 directs dynactindependent RAB-11 endosomal recycling during mitosis in Caenorhabditis elegans. Mol. Biol. Cell 20:1629-1638.

Berns, H., Humar, R., Hengerer, B., Kiefer, F., and Battegay, E. 2000. RACK1 is up-regulated in angiogenesis and human carcinomas. FASEB (Fed. Am. Soc. Exp. Biol.) J. 14:2549-2558.

Cao, X. X., Xu, J. D., Xu, J. W., Liu, X. L., Cheng, Y. Y., Li, Q. Q., Xu, Z. D., and Liu, X. P. 2009. RACK1 promotes breast carcinoma proliferation and invasion/metastasis in vitro and in vivo. Breast Cancer Res Treat. 123:375-386.

Ceci, M., Gaviraghi, C., Gorrini, C., Sala, L. A., Offenhauser, N., Marchisio, P. C., and Biffo, S. 2003. Release of eIF6 (p27BBP) from the 60S subunit allows $80 \mathrm{~S}$ ribosome assembly. Nature 426:579-584.

Chen, J. G., Ullah, H., Temple, B., Liang, J., Guo, J., Alonso, J., Ecker, J., and Jones, A. 2006. RACK1 mediates multiple hormone responsiveness and developmental processes in Arabidopsis. J. Exp. Bot. 57:26972708.

Chen, S., Lin, F., Shin, M. E., Wang, F., Shen, L., and Hamm, H. E. 2008. RACK1 regulates directional cell migration by acting on $G \beta \gamma$ at the interface with its effectors PLC $\beta$ and PI3K $\gamma$. Mol. Biol. Cell 19:39093922.

Cheon, C. L., Lee, N. G., Siddique, A. B., Bal, A. K., and Verma, D. P. 1993. Roles of plant homologs of Rab1p and Rab7p in the biogenesis of the peribacteroid membrane, a subcellular compartment formed de novo during root nodule symbiosis. EMBO (Eur. Mol. Biol. Organ.) J. 12:4125-4135

Chomczynski, P., and Sacchi, N. 1987. Single-step method of RNA isolation by acid guanidinium thiocyanate-phenol-chloroform extraction. Anal. Biochem. 162:156-159.

Crespi, M., and Frugier, F. 2008. De novo organ formation from differentiated cells: Root nodule organogenesis. Sci. Signal. 1:re11.

Ding, Y., and Oldroyd, G. E. D. 2009. Positioning the nodule, the hormone dictum. Plant Signal. Behav. 4:89-93.

Estrada-Navarrete, G., Alvarado-Affantranger, X., Olivares, J.-E., DíazCamino, C., Santana, O., Murillo, E., Guillén, G., Sánchez-Guevara, N., Acosta, J., Quinto, C., Li, D., Gresshoff, P. M., and Sánchez, F. 2006. Agrobacterium rhizogenes transformation of the Phaseolus spp.: A tool for functional genomics. Mol. Plant-Microbe Interact. 19:1385-1393.

Estrada-Navarrete, G., Alvarado-Affantranger, X., Olivares, J.-E., Guillén, G., Díaz-Camino, C., Campos, F., Quinto, C., Gresshoff, P. M., and 
Sánchez, F. 2007. Fast, efficient and reproducible genetic transformation of Phaseolus spp. by Agrobacterium rhizogenes. Nat. Protocols 2:1819-1824.

Gough, C. 2003. Rhizobium Symbiosis: Insight into Nod Dispatch Factor Receptors. Curr. Biol. 13:R973-R975.

Guo, J., and Chen, J.-G. 2008. RACK1 genes regulate plant development with unequal genetic redundancy in Arabidopsis. BMC Plant Biol. doi: 10.1186/1471-2229-8-108. Published online.

Guo, J., Wang, J., Xi, L., Huang, W. D., Liang, J., and Chen, J. G. 2009. RACK1 is a negative regulator of ABA responses in Arabidopsis. J. Exp. Bot. 60:3819-3833.

Hirsch, A. M., and Fang, Y. 1994. Plant hormones and nodulation: What's the connection? Plant Mol. Biol. 26:5-9.

Ishida, S., Takahashi, Y., and Nagata, T. 1993. Isolation of cDNA of an auxin-regulated gene encoding a $G$ protein $\beta$ subunit-like protein from tobacco BY-2 cells. Proc. Natl. Acad. Sci. U.S.A. 90:11152-11156.

Islas-Flores, T., Guillén, G., Islas-Flores, I., San Román-Roque, C., Sánchez, F., Loza-Tavera, H., Bearer, E., and Villanueva, M. A. 2009. Germination behavior, biochemical features and sequence analysis of the RACK1/arcA homolog from Phaseolus vulgaris. Physiol. Plant. 137:264-280.

Iwasaki, Y., Komano, M., Ishikawa, A., Sasaki, T., and Asahi, T. 1995. Molecular cloning and characterization of cDNA for a rice protein that contains seven repetitive segments of the Trp-Asp forty-amino-acid repeat (WD-40 repeat). Plant Cell Physiol. 3:505-510.

Kiyosue, T., and Ryan, C. 1999. The electronic plant gene register. Plant Physiol. 119:1567-1568.

Kraus, S., Gioeli, D., Vomastek, T., Gordon, V., and Weber, M. J. 2006. Receptor for activated C kinase 1 (RACK1) and Src regulate the tyrosine phosphorylation and function of the androgen receptor. Cancer Res. 66:11047-11054.

Kumagai, H., Hakoyama, T., Umehara, Y., Sato, S., Kaneko, T., Tabata, S., and Kouchi, H. 2007. Protein, IGN1, is required for persistence of nitrogen-fixing symbiosis in root nodules of Lotus japonicus. Plant Physiol. 143:1293-1305.

Kwak, J., Kim, S., Lee, S., Oh, S.-A., Byoun, C.-H., Han, J.-K., and Nam, H. 1997. cDNA clone with high similarity to a mammalian receptor for activated protein kinase C. Planta 201:245-251.

McCahill, A., Warwicker, J., Bolger, G. B., Houslay, M. D., and Yarwood, S. J. 2002. The RACK1 scaffold protein: A dynamic cog in cell response mechanisms. Mol. Pharmacol. 62:1261-1273.

McKhann, H., Frigier, F., Petrovics, G., Coba de la Peña, T., Jurkevitch, E., Brown, S., Kondorosi, E., Kondorosi, A., and Crespi, M. 1997. Cloning of a WD-repeat-containing gene from alfalfa (Medicago sativa): A role in hormone-mediated cell division? Plant Mol. Biol. 34:771-780.

Nakashima, A., Chen, L., Thao, N. P., Fujiwara, M., Wong, H. L., Kuwano, M., Umemura, K., Shirasu, K., Kawasaki, T., and Shimamoto, K. 2008. RACK1 Functions in rice innate immunity by interacting with the Rac1 immune complex. Plant Cell 20:2265-2279.

Nielsen, N., Beilinson, V., Bassüner, R., and Reverdatto, S. 2001. A G $\beta^{-}$ like protein from soybean. Physiol. Plant. 111:75-82.

Oldroyd, G. E.D., and Downie, J. A. 2008. Morphogenesis with rhizobial infection in legumes. Annu. Rev. Plant Biol. 59:519-546.

Pawlowski, K., Kunze, R., De Vries, S., and Bisseling, T. 1994. Isolation of total, poly(A) and polysomal RNA from plant tissues. Pages 1-13 in: Plant Molecular Biology Manual, S. B. Gelvin and R. A. Schilperoort, eds., Kluwer Academic Publishers, Dordrecht, The Netherlands.

Ron, D., Chen, C.-H., Caldwell, J., Jamieson, L., Orr, E., and MochlyRosen, D. 1994. Cloning of an intracellular receptor for protein kinase C: A homolog of the $\beta$ subunit of $G$ proteins. Proc. Natl. Acad. Sci. U.S.A. 91:839-843.

Saito, A., Fujii, G., Sato, Y., Gotoh, M., Sakamoto, M., Toda, G., and Hirohashi, S. 2002. Detection of genes expressed in primary colon cancers by in situ hybridisation: Overexpression of RACK 1. J. Clin. Pathol. Mol Pathol. 55:34-39.

Ullah, H., Scappini, E. L., Moon, A. F., Williams, L. V., Armstrong, D. L., and Pedersen, L. C. 2008. Structure of a signal transduction regulator, RACK1, from Arabidopsis thaliana. Protein Sci. 17:1-10.

Valdés-López, O., Huertero-Arenas, C., Ramírez, M., Girard, L., Sánchez, F., Vance, C., Reyes, J. L., and Hernández, G. 2008. Essential role of MYB transcription factor: PvPHR1 and microRNA: PvmiR399 in phosphorus-deficiency signalling in common bean roots. Plant Cell Environ. 31:1834-1843. 